\title{
Field Efficacy of Certain Plant Derivatives against the Major Sucking Pests of Brinjal Solanum melongena L.
}

\author{
S. Ayyanar ${ }^{1 *}$, C. Chinniah ${ }^{1}$, M. Kalyanasundram ${ }^{1}$, K. Balakrishnanan ${ }^{2}$ and \\ M. Muthamilan ${ }^{3}$ \\ ${ }^{1}$ Department of Agricultural Entomology, ${ }^{2}$ Department of Crop Physiology, Agricultural College \\ and Research Institute, Madurai-625104, Tamil Nadu, India \\ ${ }^{3}$ Department of Plant Pathology, Tamil Nadu Agricultural University, CBE-641003, \\ Tamil Nadu, India \\ *Corresponding author
}

\begin{tabular}{|c|c|}
\hline & A B S T R A C T \\
\hline $\begin{array}{l}\text { K e y w o r d s } \\
\text { Brinjal pests, Efficacy } \\
\text { of plant derivatives, } \\
\text { Botanical pest } \\
\text { management, Eco } \\
\text { friendly pest } \\
\text { management. }\end{array}$ & \multirow{3}{*}{$\begin{array}{l}\text { The field experiment was carried out on farmers holding at Melur block of } \\
\text { Madurai dt using the popular brinjal variety Kallampatti local during } \\
\text { summer } 2016 \text { to evaluate the efficacy of certain promising plant derivatives } \\
\text { against major sucking pests of brinjal viz., aphids, brown plant hopper, } \\
\text { thrips, whiteflies and two spotted spider mites. The overall impact of three } \\
\text { rounds of spray revealed that basil leaf extract } 10 \% \text { caused maximum } \\
\text { reduction of sucking pest population followed by Nochi leaf extract } 5 \% \text {, } \\
\text { apart from this, Neem oil } 3 \% \text { had moderate efficacy against sucking pests } \\
\text { followed by Rosemary oil } 2 \% \text {, Cashew nut shell liquid } 3 \% \text {, Garlic bulb } \\
\text { extract } 10 \% \text {. Followed by Soap nut powder extract } 10 \% \text {, Fish oil rosin soap } \\
20 \% \text {. }\end{array}$} \\
\hline Articl & \\
\hline $\begin{array}{l}\text { Accepted: } \\
\text { 29 September } 2017 \\
\text { Available Online: } \\
\text { 10 October } 2017\end{array}$ & \\
\hline
\end{tabular}

\section{Introduction}

Brinjal, Solanum melongena L. is an important vegetable crop cultivated throughout India and many other countries for its nutritious fruits. The common names are Eggplant, Aubergine, Terong, Oriental eggplant, Garden eggplant, Italian eggplant, Berenjena, Nasu, Talong, Brinjal, Melongena, Guinea squash, Apple of love, Asiatic aubergine, and poor-man's caviar, Susumber white eggplants etc. The genus Solanum belongs to the family Solanaceae (Night shade family) under the order Solanales. A small tropical perennial with attractive purple flowers, native of Africa and Asia: growing up to a height of 55 inches. Wild types can grow much longer, up to 85 inches. The leaves are pubescent and sometimes spiny. The fruits grow upto 25 inch long which may weigh up to $21 / 2 \mathrm{lb}$. and have numerous soft seeds. These fruits may be black, purple, green, white, striped and sometimes even red. Eggplant is available in several shapes, sizes and colours. This vegetable with a spongy texture is also used extensively in the Surinam Kitchen. 
However, brinjal (Solanum melongena. Linnaeus) is highly cosmopolitan and popular vegetable grown is grown in area of 0.75 million hectares with an annual production 15.6 million tons all over the country. Although area under brinjal is increasing, the year by year the average production is much lower. In Southern district of Tamil nadu Madurai district is one of the leader in production followed by Dindigal, Theni, Virudhunagar, Ramnad, Tuticorin and Kanyakumari, which are the major contributors' in terms of area and production.

The insect pest problems in brinjal are becoming more serious owing to favourable conditions such as methods of cultivation. The monoculture, adaption high yielding hybrids overlapping crops, dense cropping, excessive use of fertilizers and pesticides, continuous availability of preferred host plants are some of the major reasons for the pest outbreaks. About 142 Insect species have been recorded to infest brinjal crop.

For the initial vegetative stage the sucking pest viz., aphids, brown leafhopper, whiteflies, thrips and spider mites are the prominent important pest that causes significant yield loss.

The fruit fetches low price and become unfit for consumption. Even among sucking pests the red spider mite are the most dreadful pests, Tetranychus spp, (Tetranychidae: Acarina) often devastate the crop. Pillai et al., 1983 have reported that complex of four species of mite viz., Eutetranychus orientalis (Klein), Tetranychus cinnabarinus (Biosdual) and T.neocaledonicus (Andre) are known to heavy cause yield losses $36.8 \%$ to $83.2 \%$ on vegetable crops in India

Anitha and Nandhihalli (2008) reported that incidence of mite in summer crop commenced from $16^{\text {th }}$ Std week (2.2 mites $/ 3$ leaves) Puttasamy and Channabasavanna (1980) reported that population of mites attained a peak during May-June. The first sight of infestation by red spider mite is usually chlorotic, stippled appearance on the leaves. As the mites feed on the undersurface of leaves, they remove leaf cell contents, including chlorophyll, without which the empty cells appear whitish or bronze.

\section{Materials and Methods}

The field experiment was conducted to study the efficacy of certain selective plant derivatives against the major sucking pests of brinjal. The field experiment was laid out in a farmers holding at kallampatti village, Melur block, Madurai District which is a traditional brinjal belt. The field experiment was laid out during summer 2016, in an RBD using the variety kallampatti local (pinkish fleshy type) very popular in this traditional brinjal tract during this season. The plot size of $20 \mathrm{~m}^{2}$ was maintained with three replications each, maintaining spacing of $60 \times 60 \mathrm{~cm}$ between the planting and between rows. The soil was red loamy well suited for vegetable cultivation and the preceding crop was rice. Totally there were eleven treatments and standard check and untreated check for comparison.

Totally three rounds of sprays treatments were imposed at 15 days interval starting from 20 days after transplanting i.e after the onset of sucking pest incidence considering the ETL. Hand operated high volume sprayer (Aspee knapsack) was employed with a spray fluid of $200 \mathrm{lit} / \mathrm{ac}$. The counted on sucking pests were recorded on 1, 3, 7 and 14 days after each spray apart from pretreatment count. The mean population was worked out at for statistical analysis after square root transformation the cumulative mean was computed for calculation of percent of pests and compare the efficacy of various plant products by LSD in suppressing sucking pests viz., aphids, leafhopper, thrips, whiteflies and 
mites that occurred in the early stage of the crop growth the fruit yield was Rendell staggered by treatment wise and replication wise in $\mathrm{kg} / \mathrm{plot}$ which was later computed tonnes/ha for work out the CBR to identify the best treatment, taking into consideration the percent reduction of pest population and the percent increase of yielded over the untreated check.

Preparation of aqueous extract of botanicals

\section{Leaf extracts}

Citronella $30 \mathrm{gm}$ leaf, nochi $50 \mathrm{gm}$ and basil $10 \mathrm{gm}$ were weighed and made in to fine slurry using sufficient quantity of water. The slurry was filtered through muslin cloth and the filtrate was made up to $100 \mathrm{ml}$ with distilled water and used as the stock solution. The concentration $3 \%, 5 \%$ and $10 \%$ were prepared from the stock solution and used for further studies.

\section{Seed extracts}

Soap dried seed nut $10 \mathrm{gm}$ as powder was soaked in $100 \mathrm{ml}$ of water overnight. The slurry was filtered through muslin cloth and the filtrate was made up to $100 \mathrm{ml}$ with water and the used as the stock solution $10 \%$ and $30 \%$ concentration were prepared from the stock solution and used for further studies.

\section{Bulb extract}

The bulb extract of garlic Allium sativum was prepared by adopting the procedure suggested by Premalatha et al., (2017). Bulb purchased from herbal shop were dried under shade condition powdered and passed through a 20 mesh sieve to obtain fine and uniform powder. $10 \mathrm{gm}$ of the powder was taken in a $250 \mathrm{ml}$ conical flask and $100 \mathrm{ml}$ of water was added to it and the mixture was stirred in a mechanical shaker for about $8 \mathrm{hrs}$ and then kept at room condition for $24 \mathrm{hrs}$. The extract was then filtered through a fine muslin cloth. The filtrate was collected in a $100 \mathrm{ml}$ capacity volumetric flask and the volume was made up to $100 \mathrm{ml}$ with water. This was considered as the stock solution. From the stock solution the required concentrations were prepared by serial dilution for and further studies.

\section{Technical programme}

Season: Summer -2016

Variety: kallampatti local

Soil type: Red soil

Design: Randomizied Block Design

Plot size: $4 \times 5 \mathrm{~m}^{2}$

Spacing: $60 \times 60 \mathrm{~cm}$

Replication: Three

Treatments: 11

\section{Assessment of pest population}

\section{Aphids (Aphis gossypii Glover)}

The aphids population was assessed from three leaves per plant representing top, middle and bottom portion of the plant prior to spray and on $1^{\text {st }}, 3^{\text {rd }}, 7^{\text {th }}$ and 14 days after spray. The number of nymphs and adults were counted from each leaf. For each replication ten plants were selected at random for each treatment. The population was expressed as mean number aphids/leaf. For transformation statistical scouting for comparing the mean.

\section{White flies (Bemisia tabaci Gennadius)}

Whitefly is a notorious vector, which transmits an array of pathogen. It has piercing and sucking mouthparts and both nymphs and adults feed on under surface of the leaves causing malformation of young leaves. Whiteflies also excrete honey dew, causing development of sooty mould. The whitefly population was assessed from three leaves representing the top, middle and bottom portion of the plant apart from pretreatment 
count post treatment counts were to spray $1^{\text {st }}, 3^{\text {rd }}, 7^{\text {th }}$ and 14 days after spray. Each spray the number of nymphs and adults were counted from each leaf. Totally ten plants were selected plants at random per replication for sampling and the mean. The population was expressed as number of whiteflies/leaf

\section{Brown leaf hopper (Cestius phycitis Distant)}

The leaf hopper population was assessed from three leaves representing the top, middle and bottom portion of the plant apart from pretreatment the post treatment count were prior to treatment and $1^{\text {st }}, 3^{\text {rd }}, 7^{\text {th }}$ and 14 days after treatment. The number of nymphs and adults were counted from ten randomly selected plants replication. The population was expressed as number/leaf.

\section{Thrips (Thrips tabaci Lindermann)}

The thrips population was assessed from three leaves representing the top, middle and bottom portion of the plant selected at random. Apart pre count and post count were observed on $1^{\text {st }}, 3 \mathrm{rd}$, 7th and 14 days after each spray. The number of nymphs and adults were counted from from each leaf. For each treatment ten randomly selected plants were sampled per replication. The population was expressed as number of thrips/leaf.

\section{Two spotted spider mites (Tetranychus} urticae Koch)

The two spotted spider mite was assessed from $2 \mathrm{~cm} 2$ area of three leaves representing the top middle and bottom portion of the plant on $1^{\text {st }}, 3 \mathrm{rd}, 7$ th and $14^{\text {th }}$ days after each spray by using $10 \mathrm{X}$ hand lens. The eggs and active stages were recorded from each leaf, on ten plants selected at random per replication. The population was expressed in terms of number of mites $/ \mathrm{cm}^{2} /$ area of leaf.

\section{Yield}

The yield of brinjal fruits was recorded from plot and replication staggerdly as and when harvested and the same was weighed and recorded. The yield was computed as $\mathrm{Kg} / \mathrm{ha}$ and the increase in yield in treated plots over the untreated check was worked out to compare the efficacy of treatments and the cost benefit ratio.

\section{Statistical analysis}

The data on the pest numbers (pest population) were transformed into square root values, before analysis. Duncan (1951) Multiple Range Test (DMRT) was applied for comparing the treatment means correlation co-efficient was worked out according to Singh and Chaudhary (1979). In order to assess the interaction between treatments, the data were subjected to factorial RBD analysis and the treatment means obtained were separated by LSD (Least significant Difference)

\section{Results and Discussion}

The field investigation revealed that (Table 1). The pretreatment count of aphids ranged between 24.3 to 25.8 nos/leaf which were statistically non-significant. Among the ten plant derivatives evaluated, Basil leaf extract $10 \%$, nochi leaf extract $5 \%$, neem oil $3 \%$ recorded the maximum percent reduction of aphids 67.73, 64.94, 62.15\% respectively, which were statistically on par in their bio efficacy. Other treatments worth mentioning with moderate toxicity towards aphids are Rosemery oil 2\% (60.56\%) mortality and cashew nut shell liquid 3\% (57.77\%)which were statistically on par in terms of efficacy followed by Garlic bulb extract $10 \%$ $(52.99 \%)$ and citronella oil $3 \%(38.65 \%)$ cashew nut shell liquid 3\%(57.77\%,) respectively. The least efficacy was observed in case of soap nut $10 \%(37.05 \%)$ and FORS 
$20 \%(29.08 \%)$.

However Imidacloprid 17.8 SL@ 1.3ml/lit (standard check) was significantly superior to all the treatments evaluated with the highest percent reduction of aphid (93.79\%). Hence among the plant derivatives evaluated Tulsi leaf extract $10 \%$ and nochi leaf extract $5 \%$ and neem oil 3\% were significantly superior to all other treatments in term of their efficacy against aphids.

As for as leaf hopper (Cestius phycitus) population is concerned (Table 2) the pretreatment count did not vary among the treatments (10.1 to 11.8 nos/leaf). There was a maximum number of population reduction in basil leaf extract (Ocimum indicum) 10\% $(75.68 \%)$ followed by nochi leaf extract (Vitex nugundo) @5\% (74.77\%),neem oil (Azadirachta indica) @ 3\% (73.87\%) followed by, rosemary oil 2\% (72.07\%),Fish oil rosin soap $20 \%$ (71.17\%) followed by soapnut seed extract $10 \%$ (62.16\%), garlic bulb extract (Allium sativum) @10\% $(59.46 \%)$ and cashew nut shell liquid 3\% $(53.15 \%)$ respectively. Whereas citronella oil $3 \% \quad(44.14 \%)$ was less effective comparatively. The popular insecticide
Imidacloprid 17.8SL@1.3 ml/lit was superior to other plant derivatives (91.07\%) compared for their efficacy against leaf hopper infestation.

The pretreatment count of thrips population ranged from 17.1 to 18.5 (Table 3) per leaf. There was significant reduction of thrips population after spray due to different plant products i.e., basil leaf extract $10 \%$, Nochi leaf extract 5\% and Neem oil 3\% (76.92\%, $74.18 \%$ and $67.58 \%$ ) respectively, followed by neemoil $3 \%(67.58 \%)$ and rosemary oil 2\% (65.93\%) Fish oil rosin soap@20\% (60.99\%). However Soap nut 10\% (Sapindus marginatus) $48.90 \%$, garlic bulb extract @ 10\% $43.96 \%$ cashew nut shell liquid @ 3\% $37.36 \%$, citronella oil @3\% 32.97\% registered less than 50\% mortality. The standard check Imidacloprid 17.8SL @ 1.3 $\mathrm{ml} /$ lit was superior to other plant derivatives $(89.98 \%)$ evaluated. As for as whitefly population is concerned (Table 4) they decline gradually after 3 days of imposing treatments the maximum population reduction was noticed in Tulsi leaf extract $10 \%$ (72.15\%) followed by Nochi 5\% (71.23\%), Neem oil 3\% (67.12\%).

The following are the treatment details

\begin{tabular}{|l|l|c|c|}
\hline T. No. & Treatments & Concentration & Plant part used \\
\hline $\mathrm{T}_{1}$ & Soap nut seed extract (Sapindus marginatus) L. & $10 \%$ (Aqueous extract) & Seed \\
\hline $\mathrm{T}_{2}$ & Garlic bulb extract (Allium sativum)L. & $10 \%$ (Aqueous extract) & Bulbs \\
\hline $\mathrm{T}_{3}$ & Citronella oil (Cymbopogon nardus) L. & $3 \%$ & Leaf \\
\hline $\mathrm{T}_{4}$ & Neem oil (Azadirachta indica) L. & $3 \%$ & Seed \\
\hline $\mathrm{T}_{5}$ & Nochi leaf extract (Vitex negundo)L. & $5 \%$ (Aqueous extract) & Leaf \\
\hline $\mathrm{T}_{6}$ & Basil leaf extract (Ocimum sanctum) L. & $10 \%$ (Aqueous extract) & Leaf \\
\hline $\mathrm{T}_{7}$ & Fish oil Rosin Soap (FORS) & $20 \%$ & - \\
\hline $\mathrm{T}_{8}$ & Cashew nut shell liquid (Anacardium occidentale) L. & $3 \%$ & Nut shell \\
\hline $\mathrm{T}_{9}$ & Rosemery oil (Rosmarinus officinalis) L. & $2 \%$ & - \\
\hline $\mathrm{T}_{10}$ & Imidacloprid 17.8 SL/Propargite 57 EC @ & $0.25 \mathrm{ml} /$ lit/2ml/lit & \\
\hline $\mathrm{T}_{11}$ & Untreated check & - & - \\
\hline
\end{tabular}


Table.1 Field efficacy of certain plant derivatives against aphids (Aphis gossypii Glover) on brinjal Season - summer 2016; location - Kallam patti (Melur block)

\begin{tabular}{|c|c|c|c|c|c|c|}
\hline \multirow{2}{*}{ Treatments } & \multirow{2}{*}{$\begin{array}{c}\text { Pre } \\
\text { treatment } \\
\text { count }\end{array}$} & \multicolumn{3}{|c|}{ No. of aphids /leaf } & \multirow{2}{*}{$\begin{array}{l}\text { Cumulative } \\
\text { Mean }\end{array}$} & \multirow{2}{*}{$\begin{array}{c}\text { \% reduction } \\
\text { over } \\
\text { untreated } \\
\text { check } \\
\end{array}$} \\
\hline & & I $^{\text {st }}$ spray & II $^{\text {nd }}$ spray & III $^{\text {rd }}$ spray & & \\
\hline $\begin{array}{l}\mathrm{T}_{1} \text { - Soap nut seed extract (Sapindus marginatus) L. } \\
\text { @ } 10 \%\end{array}$ & 24.90 & $\begin{array}{l}16.31 \\
(4.04)^{\mathrm{i}}\end{array}$ & $\begin{array}{l}16.18 \\
(3.9)^{\mathrm{i}}\end{array}$ & $\begin{array}{l}15.12 \\
(3.89)^{\mathrm{i}}\end{array}$ & $\begin{array}{l}15.00 \\
(3.97)^{\mathrm{i}}\end{array}$ & 37.05 \\
\hline $\mathrm{T}_{2}$ - Garlic bulb extract (Allium sativum)L. @10\% & 25.10 & $\begin{array}{c}12.27 \\
(3.50)^{\mathrm{g}} \\
\end{array}$ & $\begin{array}{c}11.45 \\
(3.38)^{g}\end{array}$ & $\begin{array}{c}11.41 \\
(3.41)^{\mathrm{g}} \\
\end{array}$ & $\begin{array}{c}11.8 \\
(3.44)^{\mathrm{g}} \\
\end{array}$ & 52.99 \\
\hline $\mathrm{T}_{3}$ - Citronella oil (Cymbopogon nardus) L. @ 3\% & 24.30 & $\begin{array}{c}15.41 \\
(3.84)^{\mathrm{h}}\end{array}$ & $\begin{array}{c}15.35 \\
(3.92)^{\mathrm{h}}\end{array}$ & $\begin{array}{c}15.24 \\
(3.90)^{\mathrm{h}}\end{array}$ & $\begin{array}{c}15.33 \\
(3.92)^{\mathrm{h}}\end{array}$ & 38.65 \\
\hline $\mathrm{T}_{4}$ - Neem oil (Azadirachta indica) L. @ 3\% & 25.40 & $\begin{array}{c}9.75 \\
(3.07)^{\mathrm{d}}\end{array}$ & $\begin{array}{c}9.51 \\
(3.08)^{d}\end{array}$ & $\begin{array}{c}9.47 \\
(3.08)^{\mathrm{d}}\end{array}$ & $\begin{array}{c}9.57 \\
(3.08)^{d}\end{array}$ & 62.15 \\
\hline $\mathrm{T}_{5}$ - Nochi leaf extract (Vitex negundo)L. @ 5\% & 25.80 & $\begin{array}{c}9.18 \\
(3.03)^{\mathrm{c}}\end{array}$ & $\begin{array}{c}8.82 \\
(2.98)^{c}\end{array}$ & $\begin{array}{c}8.52 \\
(2.92)^{c}\end{array}$ & $\begin{array}{c}8.84 \\
(2.97)^{c}\end{array}$ & 64.94 \\
\hline $\mathrm{T}_{6}$ - Basil leaf extract (Ocimum sanctum) L. @ 10\% & 24.00 & $\begin{array}{c}8.29 \\
(2.88)^{\mathrm{b}}\end{array}$ & $\begin{array}{c}8.16 \\
(2.86)^{b}\end{array}$ & $\begin{array}{c}7.85 \\
(2.80)^{b}\end{array}$ & $\begin{array}{c}8.10 \\
(2.85)^{b}\end{array}$ & 67.73 \\
\hline $\mathrm{T}_{7}$ - Fish oil Rosin Soap (FORS) @ 20\% & 25.60 & $\begin{array}{l}18.32 \\
(4.17)^{\mathrm{j}}\end{array}$ & $\begin{array}{l}17.95 \\
(4.24)^{\mathrm{j}}\end{array}$ & $\begin{array}{l}17.19 \\
(4.15)^{\mathrm{j}}\end{array}$ & $\begin{array}{c}17.80 \\
(4.22)^{\mathrm{j}}\end{array}$ & 29.08 \\
\hline $\mathrm{T}_{8}$ - CNSL (Anacardium occidentale) L. @ 3\% & 25.00 & $\begin{array}{c}10.72 \\
(3.27)^{\mathrm{f}}\end{array}$ & $\begin{array}{c}10.63 \\
(3.22)^{\mathrm{f}}\end{array}$ & $\begin{array}{c}10.12 \\
(3.18)^{f}\end{array}$ & $\begin{array}{c}10.60 \\
(3.26)^{f}\end{array}$ & 57.77 \\
\hline T9 - Rosemeryoil (Rosmarinus officinalis) L. @ 2\% & 24.70 & $\begin{array}{c}10.25 \\
(3.15)^{\mathrm{e}}\end{array}$ & $\begin{array}{c}10.09 \\
(3.18)^{\mathrm{e}}\end{array}$ & $\begin{array}{c}9.96 \\
(3.16)^{\mathrm{e}} \\
\end{array}$ & $\begin{array}{c}9.90 \\
(3.15)^{\mathrm{e}}\end{array}$ & 60.56 \\
\hline $\mathrm{T}_{10}$ - Imidacloprid 17.8 SL @0.25 ml/lit & 25.60 & $\begin{array}{c}6.21 \\
(2.49)^{\mathrm{a}}\end{array}$ & $\begin{array}{c}2.13 \\
(1.45)^{\mathrm{a}}\end{array}$ & $\begin{array}{c}1.56 \\
(1.24)^{\mathrm{a}} \\
\end{array}$ & $\begin{array}{c}3.30 \\
(1.82)^{\mathrm{a}}\end{array}$ & 93.79 \\
\hline $\mathrm{T}_{11}$ - Untreated check & 25.10 & $\begin{array}{c}25.95 \\
(4.98)^{\mathrm{k}}\end{array}$ & $\begin{array}{c}25.38 \\
(5.04)^{\mathrm{k}}\end{array}$ & $\begin{array}{c}23.97 \\
(4.90)^{\mathrm{k}}\end{array}$ & $\begin{array}{c}25.10 \\
(5.01)^{\mathrm{k}}\end{array}$ & - \\
\hline $\begin{array}{l}\text { SEd } \\
\mathrm{CD}=0.05 \\
\mathrm{CV} \%\end{array}$ & NS* & $\begin{array}{c}0.0492 \\
0.1027 \\
1.65\end{array}$ & $\begin{array}{c}0.0343 \\
0.0716 \\
1.15\end{array}$ & $\begin{array}{c}0.0125 \\
0.0261 \\
0.80\end{array}$ & $\begin{array}{c}0.0391 \\
0.0817 \\
1.31\end{array}$ & - \\
\hline
\end{tabular}

*NS : Non significant

Each value in the mean of 3 replications

Figures in parentheses are square root transformed values

In a column, values denoted by common alphabets is/ are not significantly different by LSD at $\mathrm{P}=0.05 \%$ 
Table.2 Field efficacy of certain plant derivatives against Brown leaf hopper (Cestius phycitis Distant) on brinjal Season - summer 2016; location - Kallam patti (Melur block)

\begin{tabular}{|c|c|c|c|c|c|c|}
\hline \multirow[t]{2}{*}{ Treatments } & \multirow{2}{*}{$\begin{array}{c}\text { Pre } \\
\text { treatment } \\
\text { count }\end{array}$} & \multicolumn{3}{|c|}{ No.of brown leaf hopper/ leaf } & \multirow{2}{*}{$\begin{array}{l}\text { Cumulative } \\
\text { Mean }\end{array}$} & \multirow{2}{*}{$\begin{array}{c}\text { \% reduction } \\
\text { over } \\
\text { untreated } \\
\text { check }\end{array}$} \\
\hline & & I $^{\text {st }}$ spray & II ${ }^{\text {nd }}$ spray & III $^{\text {rd }}$ spray & & \\
\hline $\mathrm{T}_{1}$ - Soap nut seed extract (Sapindus marginatus) L. @ 10\% & 10.50 & $\begin{array}{c}4.32 \\
(2.08)^{\mathrm{g}}\end{array}$ & $\begin{array}{c}4.24 \\
(2.06)^{\mathrm{g}}\end{array}$ & $\begin{array}{c}4.04 \\
(2.01)^{\mathrm{g}}\end{array}$ & $\begin{array}{c}4.2 \\
(2.05)^{\mathrm{g}}\end{array}$ & 62.16 \\
\hline $\mathrm{T}_{2}-$ Garlic bulb extract (Allium sativum)L. @ 10\% & 11.40 & $\begin{array}{c}4.56 \\
(2.14)^{\mathrm{h}}\end{array}$ & $\begin{array}{c}4.49 \\
(2.12)^{\mathrm{h}}\end{array}$ & $\begin{array}{c}4.46 \\
(2.11)^{\mathrm{h}}\end{array}$ & $\begin{array}{c}4.5 \\
(2.12)^{\mathrm{h}}\end{array}$ & 59.46 \\
\hline $\mathrm{T}_{3}$ - Citronella oil (Cymbopogon nardus) L. @ 3\% & 11.20 & $\begin{array}{c}6.20 \\
(2.49)^{\mathrm{j}}\end{array}$ & $\begin{array}{c}6.20 \\
(2.49)^{\mathrm{j}}\end{array}$ & $\begin{array}{c}6.19 \\
(2.49)^{\mathrm{j}}\end{array}$ & $\begin{array}{c}6.2 \\
(2.49)^{\mathrm{j}}\end{array}$ & 44.14 \\
\hline $\mathrm{T}_{4}$ - Neem oil (Azadirachta indica) L. @ 3\% & 10.30 & $\begin{array}{c}3.03 \\
(1.74)^{d}\end{array}$ & $\begin{array}{c}2.87 \\
(1.69)^{\mathrm{d}}\end{array}$ & $\begin{array}{c}2.80 \\
(1.67)^{\mathrm{d}}\end{array}$ & $\begin{array}{c}2.9 \\
(1.70)^{\mathrm{d}}\end{array}$ & 73.87 \\
\hline $\mathrm{T}_{5}$ - Nochi leaf extract (Vitex negundo)L. @ 5\% & 11.20 & $\begin{array}{c}2.85 \\
(1.69)^{\mathrm{c}}\end{array}$ & $\begin{array}{c}2.82 \\
(1.68)^{\mathrm{c}}\end{array}$ & $\begin{array}{l}\text { a } 2.73 \\
(1.65)^{c}\end{array}$ & $\begin{array}{c}2.8 \\
(1.67)^{\mathrm{c}}\end{array}$ & 74.77 \\
\hline $\mathrm{T}_{6}-$ Basil leaf extract (Ocimum sanctum) L. @ 10\% & 11.80 & $\begin{array}{c}2.78 \\
(1.67)^{b}\end{array}$ & $\begin{array}{c}2.73 \\
(1.65)^{b}\end{array}$ & $\begin{array}{c}2.59 \\
(1.61)^{\mathrm{b}}\end{array}$ & $\begin{array}{c}2.7 \\
(1.64)^{b}\end{array}$ & 75.68 \\
\hline $\mathrm{T}_{7}-$ Fish oil Rosin Soap (FORS) @ 20\% & 10.60 & $\begin{array}{c}3.33 \\
(1.83)^{\mathrm{f}}\end{array}$ & $\begin{array}{c}3.17 \\
(1.78)^{\mathrm{f}}\end{array}$ & $\begin{array}{c}3.10 \\
(1.76)^{\mathrm{f}}\end{array}$ & $\begin{array}{c}3.2 \\
\left(1.79^{\mathrm{f}}\right.\end{array}$ & 71.17 \\
\hline $\mathrm{T}_{8}-\mathrm{CNSL}$ (Anacardium occidentale) L. @ 3\% & 10.10 & $\begin{array}{c}5.37 \\
(2.32)^{\mathrm{i}}\end{array}$ & $\begin{array}{c}5.24 \\
(2.29)^{\mathrm{i}}\end{array}$ & $\begin{array}{c}5.04 \\
(2.24)^{\mathrm{i}}\end{array}$ & $\begin{array}{c}5.2 \\
(2.28)^{\mathrm{i}}\end{array}$ & 53.15 \\
\hline T9 - Rosemeryoil (Rosmarinus officinalis) L. @ 2\% & 11.40 & $\begin{array}{c}3.16 \\
(1.78)^{\mathrm{e}}\end{array}$ & $\begin{array}{c}3.09 \\
(1.76)^{\mathrm{e}}\end{array}$ & $\begin{array}{c}3.06 \\
(1.75)^{\mathrm{e}} \\
\end{array}$ & $\begin{array}{c}3.1 \\
(1.76)^{\mathrm{e}}\end{array}$ & 72.07 \\
\hline $\mathrm{T}_{10}$ - Imidacloprid 17.8 SL @ 0.25 ml/lit & 11.60 & $\begin{array}{c}2.95 \\
(1.71)^{\mathrm{a}}\end{array}$ & $\begin{array}{c}2.22 \\
(1.49)^{\mathrm{a}}\end{array}$ & $\begin{array}{c}1.57 \\
(1.25)^{\mathrm{a}}\end{array}$ & $\begin{array}{c}2.24 \\
(1.48)^{\mathrm{a}}\end{array}$ & 91.07 \\
\hline $\mathrm{T}_{11}$ - Untreated check & 11.10 & $\begin{array}{c}10.90 \\
(3.30)^{\mathrm{k}}\end{array}$ & $\begin{array}{c}11.25 \\
(3.35)^{\mathrm{k}}\end{array}$ & $\begin{array}{c}11.15 \\
(3.34)^{\mathrm{k}}\end{array}$ & $\begin{array}{c}11.1 \\
(3.33)^{k}\end{array}$ & - \\
\hline $\begin{array}{l}\text { SEd } \\
\mathrm{CD}=0.05 \\
\mathrm{CV} \%\end{array}$ & NS* & $\begin{array}{c}0.0084 \\
0.0175 \\
0.72\end{array}$ & $\begin{array}{c}0.00820 \\
0.0171 \\
0.70\end{array}$ & $\begin{array}{c}0.0070 \\
0.0146 \\
0.60\end{array}$ & $\begin{array}{c}0.0070 \\
0.0146 \\
0.60\end{array}$ & - \\
\hline
\end{tabular}

*NS : Non significant

Each value in the mean of 3 replications

Figures in parentheses are square root transformed values

In a column, values denoted by common alphabets is/ are not significantly different by LSD at $\mathrm{P}=0.05 \%$ 
Table.3 Field efficacy of certain plant derivatives against Thrips (Thrips tabaci Lindermann) on brinjal Season - summer 2016; location - Kallam patti (Melur block)

\begin{tabular}{|c|c|c|c|c|c|c|}
\hline \multirow[t]{2}{*}{ Treatments } & \multirow{2}{*}{$\begin{array}{c}\text { Pre } \\
\text { treatment } \\
\text { count }\end{array}$} & \multicolumn{3}{|c|}{ No.of thrips per leaf } & \multirow{2}{*}{$\begin{array}{l}\text { Cumulative } \\
\text { Mean }\end{array}$} & \multirow{2}{*}{$\begin{array}{c}\% \\
\text { reduction 0ver } \\
\text { untreated } \\
\text { check }\end{array}$} \\
\hline & & I $^{\text {st }}$ spray & II $^{\text {nd }}$ spray & IIII $^{\text {rd }}$ spray & & \\
\hline $\begin{array}{l}\mathrm{T}_{1}-\text { Soap nut seed extract (Sapindus marginatus) L. } \\
\text { @ 10\% }\end{array}$ & 17.40 & $\begin{array}{c}9.36 \\
(3.06)^{\mathrm{g}}\end{array}$ & $\begin{array}{c}9.32 \\
(3.05)^{\mathrm{g}}\end{array}$ & $\begin{array}{c}9.23 \\
(3.04)^{\mathrm{g}}\end{array}$ & $\begin{array}{c}9.3 \\
(3.05)^{\mathrm{g}}\end{array}$ & 48.90 \\
\hline $\mathrm{T}_{2}$ - Garlic bulb extract (Allium sativum)L. @10\% & 18.50 & $\begin{array}{l}10.60 \\
(3.26)^{\mathrm{h}}\end{array}$ & $\begin{array}{l}10.10 \\
(3.18)^{\mathrm{h}}\end{array}$ & $\begin{array}{c}9.90 \\
(3.15)^{\mathrm{h}}\end{array}$ & $\begin{array}{c}10.2 \\
(3.19)^{\mathrm{h}}\end{array}$ & 43.96 \\
\hline T3 - Citronella oil (Cymbopogon nardus) L. @ 3\% & 18.40 & $\begin{array}{c}12.35 \\
(3.51)^{\mathrm{j}}\end{array}$ & $\begin{array}{c}12.16 \\
(3.49)^{\mathrm{j}}\end{array}$ & $\begin{array}{c}12.09 \\
(3.48)^{\mathrm{j}}\end{array}$ & $\begin{array}{c}12.2 \\
(3.49)^{\mathrm{j}}\end{array}$ & 32.97 \\
\hline $\mathrm{T}_{4}$ - Neem oil (Azadirachta indica) L. @ 3\% & 17.90 & $\begin{array}{c}6.09 \\
(2.47)^{\mathrm{d}}\end{array}$ & $\begin{array}{c}5.96 \\
(2.44)^{\mathrm{d}}\end{array}$ & $\begin{array}{c}5.65 \\
(2.38)^{d}\end{array}$ & $\begin{array}{c}5.9 \\
(2.43)^{\mathrm{d}}\end{array}$ & 67.58 \\
\hline $\mathrm{T}_{5}$ - Nochi leaf extract (Vitex negundo)L. @ 5\% & 18.30 & $\begin{array}{c}4.85 \\
(2.20)^{\mathrm{c}}\end{array}$ & $\begin{array}{c}4.66 \\
(2.16)^{c}\end{array}$ & $\begin{array}{c}4.59 \\
(2.14)^{c}\end{array}$ & $\begin{array}{c}4.7 \\
(2.17)^{\mathrm{c}}\end{array}$ & 74.18 \\
\hline $\mathrm{T}_{6}-$ Basil leaf extract (Ocimum sanctum) L. @ 10\% & 17.30 & $\begin{array}{c}4.23 \\
(2.06)^{b}\end{array}$ & $\begin{array}{c}4.21 \\
(2.05)^{b}\end{array}$ & $\begin{array}{c}4.16 \\
(2.04)^{b}\end{array}$ & $\begin{array}{c}4.2 \\
(2.05)^{b}\end{array}$ & 76.92 \\
\hline $\mathrm{T}_{7}$ - Fish oil Rosin Soap (FORS) @ 20\% & 18.00 & $\begin{array}{c}7.26 \\
(2.70)^{\mathrm{f}}\end{array}$ & $\begin{array}{c}7.06 \\
(2.66)^{\mathrm{f}}\end{array}$ & $\begin{array}{c}6.98 \\
(2.64)^{\mathrm{f}}\end{array}$ & $\begin{array}{c}7.1 \\
(2.66)^{f}\end{array}$ & 60.99 \\
\hline $\mathrm{T}_{8}-\mathrm{CNSL}$ (Anacardium occidentale) L. @ 3\% & 17.50 & $\begin{array}{c}11.55 \\
(3.40)^{\mathrm{i}}\end{array}$ & $\begin{array}{c}11.36 \\
(3.37)^{\mathrm{i}}\end{array}$ & $\begin{array}{c}11.29 \\
(3.36)^{\mathrm{i}}\end{array}$ & $\begin{array}{c}11.4 \\
(3.38)^{\mathrm{i}}\end{array}$ & 37.36 \\
\hline T9 - Rosemeryoil (Rosmarinus officinalis) L. @ 2\% & 18.50 & $\begin{array}{c}6.21 \\
(2.49)^{\mathrm{c}}\end{array}$ & $\begin{array}{c}6.20 \\
(2.49)^{\mathrm{d}}\end{array}$ & $\begin{array}{c}6.19 \\
(2.49)^{\mathrm{c}}\end{array}$ & $\begin{array}{c}6.2 \\
(2.49)^{d}\end{array}$ & 65.93 \\
\hline $\mathrm{T}_{10}$ - Imidacloprid 17.8 SL @ $0.25 \mathrm{ml} / \mathrm{lit}$ & 17.10 & $\begin{array}{c}2.35 \\
(1.53)^{\mathrm{a}}\end{array}$ & $\begin{array}{c}2.13 \\
(1.23)^{\mathrm{a}}\end{array}$ & $\begin{array}{c}1.97 \\
(1.40)^{\mathrm{a}}\end{array}$ & $\begin{array}{c}2.15 \\
(1.38)^{\mathrm{a}}\end{array}$ & 89.98 \\
\hline $\mathrm{T}_{11}$ - Untreated check & 18.30 & $\begin{array}{c}18.27 \\
(4.27)^{\mathrm{k}}\end{array}$ & $\begin{array}{c}18.22 \\
(4.27)^{\mathrm{k}}\end{array}$ & $\begin{array}{c}18.11 \\
(4.26)^{\mathrm{k}}\end{array}$ & $\begin{array}{c}18.2 \\
(4.27)^{\mathrm{k}}\end{array}$ & - \\
\hline $\begin{array}{l}\text { SEd } \\
\mathrm{CD}=0.05 \\
\mathrm{CV} \%\end{array}$ & $\mathrm{NS} *$ & $\begin{array}{c}0.0384 \\
0.0801 \\
1.58\end{array}$ & $\begin{array}{c}0.0294 \\
0.0614 \\
1.21\end{array}$ & $\begin{array}{c}0.0270 \\
0.0564 \\
1.11\end{array}$ & $\begin{array}{c}0.0243 \\
0.0506 \\
1.00\end{array}$ & - \\
\hline
\end{tabular}

*NS : Non significant

Each value in the mean of 3 replications

Figures in parentheses are square root transformed values

In a column, values denoted by common alphabets is/ are not significantly different by LSD at $\mathrm{P}=0.05 \%$ 
Table.4 Field efficacy of certain plant derivatives against whiteflies (Bemisia tabaci Gennadius) on brinjal Season - summer 2016; location - Kallam patti (Melur block)

\begin{tabular}{|c|c|c|c|c|c|c|}
\hline \multirow[t]{2}{*}{ Treatments } & \multirow{2}{*}{$\begin{array}{c}\text { Pre } \\
\text { treatment } \\
\text { count }\end{array}$} & \multicolumn{3}{|c|}{ No.of white flies per leaf } & \multirow{2}{*}{$\begin{array}{l}\text { Cumulative } \\
\text { Mean }\end{array}$} & \multirow{2}{*}{$\begin{array}{c}\text { \% reduction } \\
\text { over } \\
\text { untreated } \\
\text { check } \\
\end{array}$} \\
\hline & & $\mathbf{I}^{\text {st }}$ spray & II $^{\text {nd }}$ spray & III $^{\text {rd }}$ spray & & \\
\hline $\begin{array}{l}\mathrm{T}_{1}-\text { Soap nut seed extract (Sapindus marginatus) L. } \\
\text { @ 10\% }\end{array}$ & 22.10 & $\begin{array}{l}14.93 \\
(3.86)^{\mathrm{h}}\end{array}$ & $\begin{array}{l}14.27 \\
(3.78)^{\mathrm{h}}\end{array}$ & $\begin{array}{l}14.00 \\
(3.79)^{h}\end{array}$ & $\begin{array}{c}14.4 \\
(3.79)^{\mathrm{h}}\end{array}$ & 34.25 \\
\hline $\mathrm{T}_{2}-$ Garlic bulb extract (Allium sativum)L. @ 10\% & 20.50 & $\begin{array}{c}14.64 \\
(3.83)^{\mathrm{g}}\end{array}$ & $\begin{array}{c}14.35 \\
(3.79)^{\mathrm{g}}\end{array}$ & $\begin{array}{l}13.62 \\
(3.69)^{\mathrm{g}}\end{array}$ & $\begin{array}{c}14.2 \\
(3.77)^{\mathrm{g}}\end{array}$ & 35.16 \\
\hline $\mathrm{T}_{3}$ - Citronella oil (Cymbopogon nardus) L. @ 3\% & 22.50 & $\begin{array}{c}15.36 \\
(3.92)^{\mathrm{j}}\end{array}$ & $\begin{array}{c}15.32 \\
(3.91)^{\mathrm{j}}\end{array}$ & $\begin{array}{c}15.22 \\
(3.90)^{\mathrm{j}}\end{array}$ & $\begin{array}{c}15.3 \\
(3.91)^{\mathrm{j}}\end{array}$ & 30.14 \\
\hline $\mathrm{T}_{4}$ - Neem oil (Azadirachta indica) L. @ 3\% & 19.90 & $\begin{array}{c}7.26 \\
(2.69)^{\mathrm{d}}\end{array}$ & $\begin{array}{c}7.22 \\
(2.69)^{\mathrm{d}}\end{array}$ & $\begin{array}{c}7.12 \\
(2.67)^{\mathrm{d}}\end{array}$ & $\begin{array}{c}7.2 \\
(2.68)^{\mathrm{d}}\end{array}$ & 67.12 \\
\hline $\mathrm{T}_{5}$ - Nochi leaf extract (Vitex negundo)L. @ 5\% & 22.40 & $\begin{array}{l}6.39 \\
(2.53)^{\mathrm{c}}\end{array}$ & $\begin{array}{c}6.33 \\
(2.52)^{\mathrm{c}}\end{array}$ & $\begin{array}{c}6.19 \\
(2.49)^{\mathrm{c}}\end{array}$ & $\begin{array}{c}6.3 \\
(2.51)^{\mathrm{c}}\end{array}$ & 71.23 \\
\hline T6 - Basil leaf extract (Ocimum sanctum) L. @10\% & 20.70 & $\begin{array}{l}6.40 \\
(2.53)^{\mathrm{b}}\end{array}$ & $\begin{array}{c}6.03 \\
(2.45)^{b}\end{array}$ & $\begin{array}{c}5.88 \\
(2.42)^{b}\end{array}$ & $\begin{array}{c}6.1 \\
(2.47)^{b}\end{array}$ & 72.15 \\
\hline $\mathrm{T}_{7}$ - Fish oil Rosin Soap (FORS) @ 20\% & 21.50 & $\begin{array}{l}14.30 \\
(3.73)^{f}\end{array}$ & $\begin{array}{l}13.80 \\
(3.73)^{\mathrm{f}}\end{array}$ & $\begin{array}{l}13.60 \\
(3.69)^{\mathrm{f}}\end{array}$ & $\begin{array}{c}13.9 \\
(3.73)^{\mathrm{f}}\end{array}$ & 36.53 \\
\hline $\mathrm{T}_{8}$ - CNSL (Anacardium occidentale) L. @ 3\% & 22.80 & $\begin{array}{c}15.39 \\
(3.92)^{\mathrm{i}}\end{array}$ & $\begin{array}{c}15.03 \\
(3.92)^{\mathrm{i}}\end{array}$ & $\begin{array}{c}14.88 \\
(3.86)^{\mathrm{i}}\end{array}$ & $\begin{array}{c}15.1 \\
(3.89)^{\mathrm{i}}\end{array}$ & 31.05 \\
\hline T9 - Rosemeryoil (Rosmarinus officinalis) L. @ 2\% & 21.00 & $\begin{array}{c}10.41 \\
(3.23)^{\mathrm{e}}\end{array}$ & $\begin{array}{c}10.20 \\
(3.19)^{\mathrm{e}}\end{array}$ & $\begin{array}{c}9.69 \\
(3.11)^{\mathrm{e}}\end{array}$ & $\begin{array}{c}10.1 \\
(3.18)^{\mathrm{e}}\end{array}$ & 53.88 \\
\hline $\mathrm{T}_{10}$ - Imidacloprid 17.8 SL @ 0.25 ml/lit & 22.50 & $\begin{array}{c}2.52 \\
(1.59)^{\mathrm{a}}\end{array}$ & $\begin{array}{c}2.12 \\
(1.31)^{\mathrm{a}}\end{array}$ & $\begin{array}{c}1.33 \\
(1.15)^{\mathrm{a}}\end{array}$ & $\begin{array}{c}1.99 \\
(1.23)^{\mathrm{a}}\end{array}$ & 91.03 \\
\hline $\mathrm{T}_{11}-$ Untreated check & 20.60 & $\begin{array}{l}22.38 \\
(4.73)^{\mathrm{k}}\end{array}$ & $\begin{array}{l}21.78 \\
(4.67)^{\mathrm{k}}\end{array}$ & $\begin{array}{l}21.54 \\
(4.64)^{\mathrm{k}}\end{array}$ & $\begin{array}{c}21.7 \\
(4.68)^{\mathrm{k}}\end{array}$ & - \\
\hline $\begin{array}{l}\text { SEd } \\
\mathrm{CD}=0.05 \\
\mathrm{CV} \%\end{array}$ & NS* & $\begin{array}{c}0.0465 \\
0.0970 \\
1.63 \\
\end{array}$ & $\begin{array}{c}0.0374 \\
0.0780 \\
1.30\end{array}$ & $\begin{array}{c}0.0366 \\
0.0763 \\
1.27\end{array}$ & $\begin{array}{c}0.0384 \\
0.0800 \\
1.34 \\
\end{array}$ & - \\
\hline
\end{tabular}

*NS : Non significant

Each value in the mean of 3 replications

Figures in parentheses are square root transformed values

In a column, values denoted by common alphabets is/ are not significantly different by $\mathrm{LSD}$ at $\mathrm{P}=0.05 \%$ 
Table.5 Field efficacy of certain plant derivatives against two spotted spider mites (Tetranychus urticae Koch) on brinjal Season Summer 2016; location - Kallam patti (Melur block)

\begin{tabular}{|c|c|c|c|c|c|c|}
\hline \multirow[t]{2}{*}{ Treatments } & \multirow{2}{*}{$\begin{array}{c}\text { Pre } \\
\text { treatment } \\
\text { count }\end{array}$} & \multicolumn{3}{|c|}{ No.of two spotted spider mites/ $\mathrm{cm}^{2} /$ leaf } & \multirow{2}{*}{$\begin{array}{l}\text { Cumulative } \\
\text { Mean }\end{array}$} & \multirow{2}{*}{$\begin{array}{c}\% \\
\text { reduction } \\
\text { over } \\
\text { untreated } \\
\text { check }\end{array}$} \\
\hline & & $\mathbf{I}^{\text {st }}$ spray & II $^{\text {nd }}$ spray & III $^{\text {rd }}$ spray & & \\
\hline $\begin{array}{l}\mathrm{T}_{1} \text { - Soap nut seed extract (Sapindus marginatus) L. } \\
\text { @ } 10 \%\end{array}$ & 15.50 & $\begin{array}{c}9.41 \\
(3.07)^{\mathrm{f}}\end{array}$ & $\begin{array}{l}9.15 \\
(3.02)^{\mathrm{f}}\end{array}$ & $\begin{array}{c}9.04 \\
(3.01)^{\mathrm{f}}\end{array}$ & $\begin{array}{c}9.20 \\
(3.03)^{\mathrm{f}}\end{array}$ & 39.47 \\
\hline $\mathrm{T}_{2}$ - Garlic bulb extract (Allium sativum)L. @ 10\% & 14.70 & $\begin{array}{c}10.34 \\
(3.21)^{\mathrm{g}} \\
\end{array}$ & $\begin{array}{c}9.79 \\
(3.13)^{\mathrm{g}} \\
\end{array}$ & $\begin{array}{c}9.57 \\
(3.09)^{\mathrm{g}} \\
\end{array}$ & $\begin{array}{c}9.90 \\
(3.15)^{\mathrm{g}} \\
\end{array}$ & 34.87 \\
\hline T 3 - Citronella oil (Cymbopogon nardus) L. @ 3\% & 15.10 & $\begin{array}{c}11.08 \\
(3.33)^{\mathrm{j}} \\
\end{array}$ & $\begin{array}{c}10.96 \\
(3.31)^{\mathrm{j}} \\
\end{array}$ & $\begin{array}{r}10.66 \\
(3.26)^{j} \\
\end{array}$ & $\begin{array}{c}10.90 \\
(3.30)^{j} \\
\end{array}$ & 28.29 \\
\hline $\mathrm{T}_{4}$ - Neem oil (Azadirachta indica) L. @ 3\% & 14.60 & $\begin{array}{c}8.55 \\
(2.92)^{\mathrm{e}}\end{array}$ & $\begin{array}{c}8.11 \\
(2.85)^{\mathrm{e}}\end{array}$ & $\begin{array}{c}7.94 \\
(2.82)^{\mathrm{e}}\end{array}$ & $\begin{array}{c}8.20 \\
(2.86)^{\mathrm{e}}\end{array}$ & 46.05 \\
\hline T 5 - Nochi leaf extract (Vitex negundo)L. @ 5\% & 15.30 & $\begin{array}{c}5.24 \\
(2.29)^{\mathrm{c}} \\
\end{array}$ & $\begin{array}{c}5.06 \\
(2.25)^{\mathrm{c}} \\
\end{array}$ & $\begin{array}{c}4.99 \\
(2.23)^{\mathrm{c}} \\
\end{array}$ & $\begin{array}{c}5.10 \\
(2.26)^{\mathrm{c}} \\
\end{array}$ & 66.45 \\
\hline T6 - Basil leaf extract (Ocimum sanctum) L. @10\% & 14.90 & $\begin{array}{c}4.93 \\
(2.22)^{\mathrm{b}}\end{array}$ & $\begin{array}{c}4.87 \\
(2.21)^{b}\end{array}$ & $\begin{array}{c}4.89 \\
(2.21)^{\mathrm{b}}\end{array}$ & $\begin{array}{c}4.90 \\
(2.21)^{b}\end{array}$ & 67.76 \\
\hline $\mathrm{T}_{7}$ - Fish oil Rosin Soap (FORS) @ 20\% & 14.40 & $\begin{array}{c}10.26 \\
(3.20)^{\mathrm{h}}\end{array}$ & $\begin{array}{c}10.06 \\
(3.17)^{\mathrm{h}}\end{array}$ & $\begin{array}{c}9.98 \\
(3.16)^{\mathrm{h}}\end{array}$ & $\begin{array}{c}10.10 \\
(3.18)^{\mathrm{h}}\end{array}$ & 33.55 \\
\hline $\mathrm{T}_{8}-\mathrm{CNSL}$ (Anacardium occidentale) L. @ 3\% & 15.10 & $\begin{array}{c}10.38 \\
(3.22)^{\mathrm{i}}\end{array}$ & $\begin{array}{c}10.26 \\
(3.20)^{\mathrm{i}}\end{array}$ & $\begin{array}{c}9.96 \\
(3.16)^{\mathrm{i}}\end{array}$ & $\begin{array}{c}10.20 \\
(3.19)^{\mathrm{i}}\end{array}$ & 32.89 \\
\hline T9 - Rosemeryoil (Rosmarinus officinalis) L. @ 2\% & 15.50 & $\begin{array}{c}5.96 \\
(2.44)^{\mathrm{d}}\end{array}$ & $\begin{array}{c}5.88 \\
(2.43)^{\mathrm{d}}\end{array}$ & $\begin{array}{l}5.85 \\
(2.42)^{\mathrm{d}}\end{array}$ & $\begin{array}{c}5.90 \\
(2.43)^{\mathrm{d}}\end{array}$ & 61.18 \\
\hline $\mathrm{T}_{10}$ - Imidacloprid 17.8 SL @ 0.25 ml/lit & 13.90 & $\begin{array}{c}1.83 \\
(1.35)^{\mathrm{a}}\end{array}$ & $\begin{array}{c}1.33 \\
(1.15)^{\mathrm{a}}\end{array}$ & $\begin{array}{c}1.03 \\
(1.01)^{\mathrm{a}}\end{array}$ & $\begin{array}{c}1.39 \\
(1.17)^{\mathrm{a}}\end{array}$ & 89.96 \\
\hline $\mathrm{T}_{11}$ - Untreated check & 15.30 & $\begin{array}{c}15.25 \\
(3.90)^{\mathrm{k}}\end{array}$ & $\begin{array}{c}15.22 \\
(3.90)^{\mathrm{k}}\end{array}$ & $\begin{array}{c}15.14 \\
(3.89)^{\mathrm{k}}\end{array}$ & $\begin{array}{c}15.20 \\
(3.90)^{\mathrm{k}}\end{array}$ & - \\
\hline $\begin{array}{l}\text { SEd } \\
\mathrm{CD}=0.05 \\
\mathrm{CV} \%\end{array}$ & NS* & $\begin{array}{c}0.0330 \\
0.0688 \\
1.35\end{array}$ & $\begin{array}{c}0.0309 \\
0.0645 \\
1.27\end{array}$ & $\begin{array}{c}0.0253 \\
0.0528 \\
1.04\end{array}$ & $\begin{array}{c}0.0262 \\
0.0547 \\
1.07\end{array}$ & \\
\hline
\end{tabular}

*NS : Non Significant

Each value in the mean of 3 replications

Figures in parentheses are square root transformed values

In a column values denoted by common alphabets is/ are not significantly different by LSD at $\mathrm{P}=0.005$ 


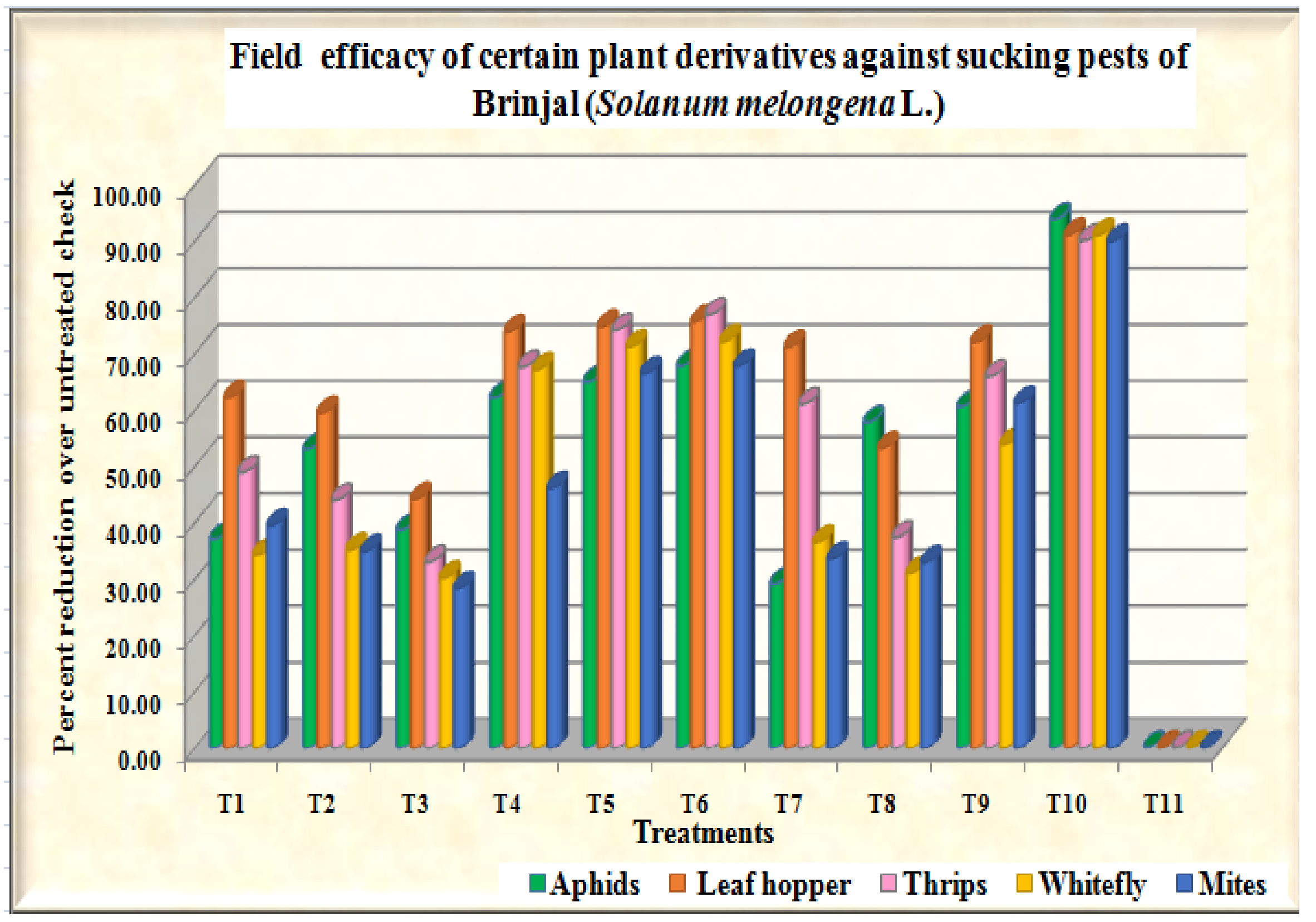


Other botanicals with mentioning are Rosemery oil 2\% (53.88) Garlic bulb extract $10 \%(35.16 \%)$ Cashew nut shell liquid 3\% $(31.05 \%)$ and Soapnut 10\% (34.25\%). However Cashew nut shell liquid $3 \%$ and Citronella oil 3\% recorded the least percentage mortality (31.05\%).

However the standard check Imidacloprid 17.8 SL @ $1.3 \mathrm{ml} /$ lit was significantly superior to all other treatments with the highest reduction $91.03 \%$. Hence among the plant derivatives tested Tulsi leaf extract 10\%, Notchi leaf extract 5\% and Neem oil 3\% exhibited promising efficacy against whiteflies.

With reference to spidermite incidence (Table 5) the pretreatment population varied from 13.9 to $15.5 \mathrm{Nos} / \mathrm{cm}^{2} / \mathrm{leaf}$. Among the plant derivatives tested against spider mites in brinjal ecosystem, after three rounds of foliar spray, the percentage reduction of mites was more in Tulsi leaf extract 10\% (67.76\%), nochi leaf extract 5\% (66.45\%) and rosemary oil $2 \%(61.18 \%)$ followed by neem oil $3 \%$ $(46.05 \%)$ all other botanicals were less effective recording less than $40 \%$ mortality over untreated check.

However the treated check propargite 57 EC (a) 2ml/lit recorded the highest percent mortality of $89.96 \%$ over untreated check. Hence tulsi, notchi and neem oil may be the best suited to be part of IPM component to suppress the population of sucking pests in brinjal ecosystem.

Aphid, Aphis gossypii Glover population recorded after first spray indicated that it was significantly reduced in all the plots treated with botanicals in comparison to untreated check. Neem oil @ 3\% proved significantly superior over rest of the botanicals. Superiority of the neem oil in suppressing the aphid population was also revealed in second spray. Plots treated with neem oil @ 3\% exhibited significantly less number of aphids than rest of the botanicals. It was suppressed significantly in plots treated with these botanicals over untreated check. The effectiveness of neem oil against sucking pests in present study is in accordance with the findings of Sardana et al., (2004) who advocated sprays of neem oil @ 3\% for effective management of insects pests of eggplant.The report of Srinivasa and Babu (2000) also have vouched that whiteflies Bemisia tabaci (Genn.) population could be effectively suppressed by using neem oil. Which is a clear evidence to support the present study that tulsi, notchi and are effective against whiteflies.This findings are also supported by Srinivasan and Babu (2000) and Rosaiah (2001) population of leafhopper, Amarasca biguttula biguttula (Ishida) after first spray was found to a minimum level (4.65 hoppers/leaf) in NSKE followed by neem oil (5.07) and custard apple leaf extract (5.12). Excellent performance of NSKE, neem oil and custard apple leaf extract was also observed after two sprays. Pooled data also indicated that the plots treated with NSKE registered minimum (4.61 hoppers/leaf) followed by neem oil (5.07). These botanicals found to be more or less equally effective against hoppers and registered significantly least population of the pest than untreated check.

This findings is in confirming with the findings of Belmain et al., (2001) who also confirmed that vitex negundo @ 2\% recorded the highest mortality with strong repellency from $(77.11 \%)$.The observation involving effect of plant extract sucking pests. It was found that vitex negundo leaf extract also registred maximum mortality against rice pests.

Nzanza et al., (2011) revealed that neem oil @3\% and wild garlic bulb extract $10 \%$ have 
insecticidal properties to maintain lower population densities of whitefly and aphid on and all parts of neem contain bitter compound (Van der Nat et al., 1991; Chawla et al., 1995) has prooved that the neem bitter has antifeedant effect which can interfere with hormonal processes in insect (Schmutterer, 1990; Ascher, 1993; Boeke et al., 2004). Similarly, wild garlic bulbs possess such as sacrid volatilic oil and sulpho -oxides derived from allicin, responsible for antifeedent, repellent and toxicant properties against various pests (vijayalakshmi et al., 1996; Dhana Lakshmi, 2006). However, the fact that the population densities of whitefly and aphid increased over time throughout the season regardless of the treatment applied suggested that neem and wild garlic could possibly have had a repellent effect on the two insects. Differences in the numbers of whiteflies and aphids in winter and summer were possibly due to seasonal variations.

Gajalakshmi, et al., 2016 also prooved that aqueous extract of Sapindus marginatus to be very effective. Among the botanicals tested Sapindus marginatus was found to have the highest acaricidal activity at $10 \%$ with the mortality of $93.33,95.83$ and $99.17 \%$ after 24, 48 and 72 hours after treatment. These results are in accordance with the study of Gengiahi et al., 2011 who reported that the saponin fraction of Dodomaea viseose caused 80\% mortality of Tetranychus urtiicae.

\section{References}

Anitha, K.R., and B.S. Nandihalli.2008. Seasonal incidence of sucking pests in okra ecosystem, karnataka J. of Agric.sci., 21(1): 137-138.

Ascher, K.R.S., 1993. Non-conventional insecticidal effect of pesticides available from the neem tree, Azadirachta indica. Arch. Insect biochem. 22:433-449.

Belmain, S.R., et al., 2001. Insecticidal and vertebrate toxicity associated with Ethanobotanical used as post -harvest protectants Ghana. Food Chemistry toxicology, 39: 287-291.

Boeke, S.J., M.G, Boersma, G.M, Alink van Loon JJA, Van Huis A, M. Dicke, and MCM Rietjens 2004. Safety evaluation of neem (Azadirachta indica) derived pesticides. J.Ethanopharmacol.94:2541.

Chawla, A.A., M. Kumar bansal.1995. Chemical constituents and biological activity of neem: a revirew Indain Drugs 32: 57-64.

Dhanalakshmi, D.N., 2006. Studies on storability of indigenous material and their utilization on okra sucking pests M.Sc (agri) thesis, University of Agricultural sciences, Dharwad.

Gajalakshmi, M., S. JayaRani and K.Ramaraju. 2016. Compatability of entomopathogenic fungi Beauveria bassiana with plant extracts evaluated against okra two spotted spider mite, Tetranychus urticae Koch, Indian journal of Entomology 78(3): 219-222.

Nzanza, B.D., Marias and P.Soundy.2011. Response of tomato (Solanum lycopersicum L.) to nursery inoculation with Trichoderma harzianum and arbuscular mycorrhizal fungi under field condition Acta, Agr, scand B.Sp:1-8.

Pillai, K.S., and M.S Palanoswami.1983. Economics on the control of spider mites on cassava. Entomon, 8: 373-375.

Premalatha et al., 2017. Evaluation of essential oils against two spotted spider mites, Tetranychus urticae on tomato, Ann.Pl. Protec.Sci.25 (1): 6-11.

Puttaswami, M., and G.P. Channabasavanna. 1980. Influence of host plants on the development, fecundity and longevity of Tetranychus ludeni Zacher (Acri: Tetranychidae). Indian J.Acarol.5: 8084.

Rosaiah, B., 2001. Evaluation of different 
botanicals against pest complex of brinjal. Pestology, 25(4):14-16.

Sardana, H.R., S. Arora and L.N.Kadu.2004. Development and validation of adaptable IPM in eggplant (Solanum melongena L.) in a farmer's participatory approach Indian, J.Pl, Protec., 32(1):123-128.

Schmutterer, H., 1990. Properties and potential for natural pesticides from the tree, Azadirachta indica. Annu.Rev.Entomol. 35:271-297.

Srinivasan, G., and PC.S.Babu.2000. Comparative efficacy of neem products against brinjal leaf hopper, Amrasca biguttula biguttula. Indain J. Entomol., 62(1):22-23.

Srinivasan, G., and PC.S.Babu.2000. Field evaluation of neem products against whitefly, Bemisia tabaci (Genn.) of brinjal Ann.Pl.Protec.Sci, 9(1):49-53.

Vander Nat, J.M., W.G. Vander Sluis De Silva KTD and R.P. Labadie.1991. Ethanopharmacognostical survey of Azadirachta indica A.Juss (Meliaceae) J.Ethanopharmacol.35:1-24.

Vijayalakshmi, K., B. Subashini and V.K, Shivani. 1996. Plant in pest control Garlic and Onion. Centre for Indian knowledge system, Chennai, pp: 1-20.

\section{How to cite this article:}

Ayyanar, S., C. Chinniah, M. Kalyanasundram, K. Balakrishnanan and Muthamilan, M. 2017. Field Efficacy of Certain Plant Derivatives against the Major Sucking Pests of Brinjal Solanum melongena L. Int.J.Curr.Microbiol.App.Sci. 6(10): 3678-3691. doi: https://doi.org/10.20546/ijcmas.2017.610.432 